\title{
Tumor BRCA testing in ovarian cancer and EQA scheme: our experience of a critical evaluation
}

\author{
Elisa De Paolis ${ }^{1} \cdot$ Paola Concolino $^{1} \cdot$ Maria Elisabetta Onori ${ }^{1} \cdot$ Concetta Santonocito $^{1}$ - Claudia Marchetti ${ }^{2,3}$. \\ Anna Fagotti ${ }^{2,3} \cdot$ Giovanni Scambia $^{2,3} \cdot$ Andrea Urbani $^{1,3} \cdot$ Angelo Minucci $^{1,4}$ (I)
}

Received: 17 August 2021 / Accepted: 5 October 2021 / Published online: 13 October 2021

(c) The Author(s) 2021

\begin{abstract}
Next generation sequencing (NGS) is a widespread molecular biology method integrated into clinical practice to detect genetic variants, for diagnostic and prognostic purposes. The scheduled external quality assessments (EQA) is integral part of clinical molecular laboratory quality assurance. The EQA provides an efficient system to compare analytic test performances among different laboratories, which is essential to evaluate consistency of molecular test. EQA failures demands targeted corrective action plans. In this context, the complexity of the NGS techniques requires careful and continuous quality control procedures. We report a tumor $B R C A 1 / 2(t B R C A)$ testing benchmark discrepancy provided by the European Molecular Genetics Quality Network in our laboratory during a round of EQA for somatic mutation testing of $B R C A$ genes in relation to ovarian cancer. The critical analysis emerging from the $t B R C A$ EQA is presented. We underline that harmonization processes are still required for the EQA in the molecular biology field, especially if applied to the evaluation of methods characterized by high complexity.
\end{abstract}

Keywords Next generation sequencing $\cdot$ Tumor $B R C A$ testing $\cdot$ Ovarian cancer $\cdot$ EMQN $\cdot$ EQA $\cdot$ Allelic dropout

$\begin{array}{ll}\text { Abbreviations } \\ \text { NGS } & \text { Next generation sequencing } \\ \text { EQA } & \text { External quality assessment } \\ \text { WT } & \text { Wild-type } \\ \text { FFPE } & \text { Formalin-fixed paraffin-embedded } \\ \text { tBRCA } & \text { Tumor BRCA1/2 } \\ \text { MGDUnit } & \text { Molecular and Genomic Diagnostics Unit } \\ \text { EMQN } & \text { European Molecular Genetics Quality } \\ & \text { Network } \\ \text { ADO } & \text { Allelic dropout } \\ \text { ISO } & \text { International Organization for Standardization }\end{array}$

Angelo Minucci

angelo.minucci@policlinicogemelli.it

1 Molecular and Genomic Diagnostics Unit (MGDUnit), Fondazione Policlinico Universitario A. Gemelli IRCCS, Rome, Italy

2 Division of Oncological Gynecology, Department of Women's and Children's Health, Fondazione Policlinico Universitario A. Gemelli IRCCS, Rome, Italy

3 Università Cattolica del Sacro Cuore, Rome, Italy

4 Molecular and Genomic Diagnostics Unit (MGDUnit), Fondazione Policlinico Universitario Agostino Gemelli IRCCS, Università Cattolica del Sacro Cuore, Rome, Italy

\section{Introduction}

Next generation sequencing (NGS) are beginning to replace traditional molecular techniques [1]. Giving the implementation of NGS in clinical settings, best practice guidelines are now available to introduce quality management processes. Quality management includes policies established with the aim to provide accurate laboratory test, as internal quality control and external quality assessment (EQA) programs. In particular, EQA involves the examination of the laboratory procedures by a third-party accreditation process, and are available for the majority of well-established technologies [2]. It is an inter-laboratory comparison that may extend throughout all phases of a testing cycle including interpretation of results [3]. EQA provides the examination of different types of external samples depending on the scheme version. For the germline scheme, laboratories generally receive genomic DNA extracted from cell lines. In somatic schemes, genetically engineered samples are generally provided. These artificial controls can be obtained by homogenously mixing mutant versus wild-type (WT) cell lines at defined allelic ratios, which closely mimics the formalinfixed paraffin-embedded (FFPE) tissue block [4]. 


\section{Tumor BRCA testing in ovarian cancer and EQA scheme: experience in our Molecular and Genomic Diagnostics Unit}

In order to evaluate tumor $B R C A 1 / 2(t B R C A)$ testing performed in our Molecular and Genomic Diagnostics Unit (MGDUnit) as a predictive biomarker of response to platinum-based-chemotherapy and PARP-inhibitors in ovarian cancer patients [5], we recently joined the disease-based Somatic Ovarian 2020 scheme of the European Molecular Genetics Quality Network (EMQN) [6]. The EMQN offers disease-specific and method-based EQA schemes when participating once a year to three different samples per EQA exercise. Participants are assessed on their ability to correctly genotype, interpret, and report the molecular results using their usual laboratory report format [2].

Our MGDUnit routinely assesses the BRCA molecular status using the amplicon-based library preparation kit Devyser BRCA (Devyser, Stockholm, Sweden) on Illumina MiSeq NGS platform [7, 8].

After uploading the EMQN scheme results, a discrepancy emerged between our BRCA genotyping and those expected in one of the samples provided by the scheme.

The validated sample genotype declared the presence of the BRCA1 NG_005905.2 (NM_007294.4):c.68_69del (rs80357914, p.(Glu23ValfsTer17)), variant with an expected variant allele frequency of $20 \%$. Our NGS analysis failed to identify the presence of this variant in the sample.

Despite this evidence, we received an EMQN communication that explains the observed controversial result. The artificial FFPE tumor sample provided contained a cell line engineered to generate the BRCAl c.68_69del variant. The engineering process used to generate the variant (undertaken by Horizon Discovery, Cambridge, UK), left a small piece of artificial DNA (the "engineering scar") of approximately $90 \mathrm{bp}$ in the intron upstream of the variant. The insertion site was positioned at the BRCAl c. $80+48_{-}$ c. $80+49$ site and caused the mis-priming of a PCR primer used by the Deyvser BRCA kit (Devyser AB, Stockholm, Sweden). Consequently, this engineering "scar" meant that this kit was unable to detect the BRCA1 c.68_69del variant for any laboratory using. This could potentially lead a laboratory to fail the EQA scheme; however, this did not occur because EMQN had detected the error during their assessment process and no laboratory using this kit was penalized. The technology used to make this artificial material has subsequently been superseded by the use of CRISPR system, which does not leave an engineering "scar".

\section{Question: Why did the Devyser kit not identify the c.68_69del variant in the EQA sample?}

Taking into account that poor performance in the EQA represents a relevant alert, we decided to explore the discrepancy experienced in the EMQN exercise. In particular, we reported the experimental workflow adopted to evaluate the EMQN BRCAl genotype via alternative methods considering the technical information about the artificially development of the external sample. We undertook this effort to exclude that the failure in the identification of the EMQN $B R C A 1$ variant, attributable to the analytical incompatibility of the EQA scheme and the Devyser kit, cannot occur in human tissue samples in an appropriate clinical setting.

The $c .68 \_69 d e l$ variant falls into the exon 2 of the BRCAI gene. The Devyser $B R C A$ kit amplifies this region via two different amplicons, which are only partially overlapping. The BRCA1_E2-1 amplicon has the sense primer that matches the region including the insertion site $c .80+48_{-}$ c. $80+49$, precluding its effective amplification (Fig. 1). Because the NGS results were obtained only by the WT allele, as a consequence of the allelic dropout (ADO), we

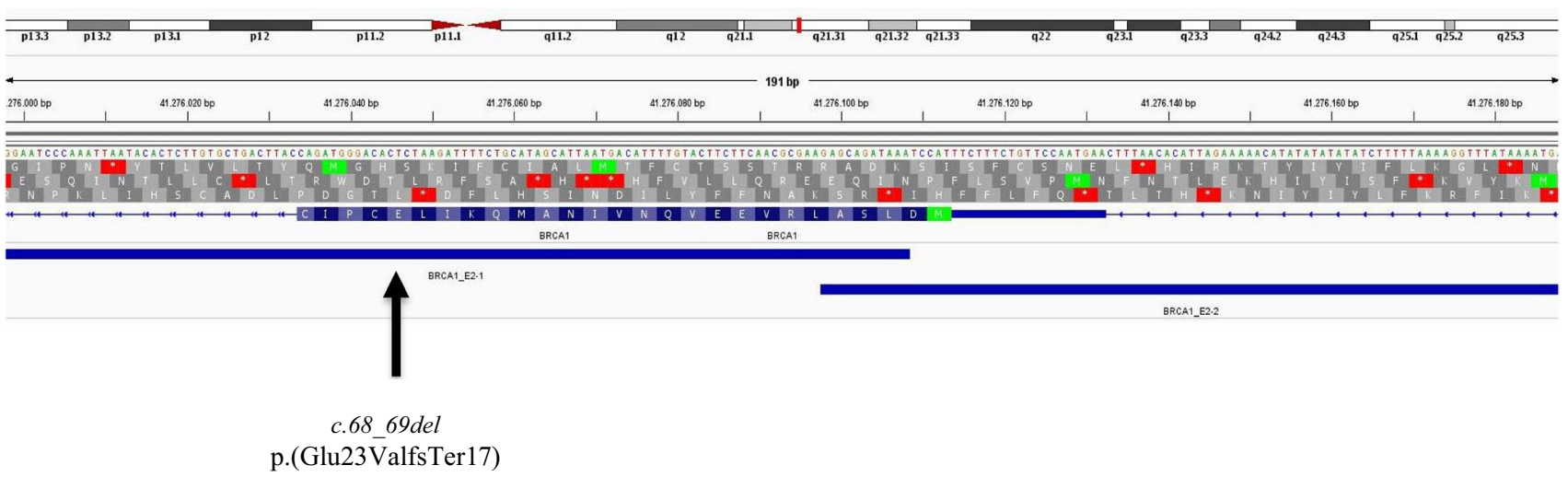

Fig. 1 Integrative genomics viewer (IGV) visualizations of the BRCAl sequence surrounding the region of interest (Human hg19) and containing the two partially overlapping Devyser amplicons BRCA1_E2-1 and BRCA1_E2-2 
missed the sequencing information regarding the $c .68$ 69 del allele. We decided to better understand the type of molecular lesion affecting the specific $B R C A 1$ region. We firstly designed ad hoc primer pair including the $c .80+48 \_c .80+49$ site (Fig. 2). Sanger sequencing of this PCR product highlighted again a WT sequence demonstrating that the engineering extends beyond the insertion site (at least up to the antisense primer). Therefore, this molecular alteration cannot occur in extracted human genomic DNA samples. However, since some $B R C A 1$ polymorphisms are reported in the $c .80+48 \_c .80+49$ position (e.g. $c .80+48 C>T$, rs200513210, and $c .80+49 C>G$ variants), the possibility of a failure in the amplification of this region using the Devyser BRCA kit remains open. Certainly, the minor allele frequency of these polymorphisms seems to be very low and they are never reported in linkage with the c.68_69del variant.

\section{Results}

\section{Identification of the c.68_69del variant by alternative PCR/NGS approach}

Since the Devyser kit was unable to identify the c.68_69del variant, we investigated and confirmed the presence of the declared BRCAl variant using an alternative PCR/ NGS molecular approach. In particular, we adopted the Hereditary Cancer Solution CE-IVD kit (SOPHiA Genetics, Saint-Sulpice, Switzerland), a NGS capture-based target enrichment of 26 cancer related genes (ATM, APC, BARD1, BRCA1, BRCA2, BRIP1, CDH1, CHEK2, EPCAM, FAM175A, MLH1, MRE11A, MSH2, MSH6, MUTYH, NBN, PALB2, PIK3CA, PMS2, PMS2C, PTEN, RAD50, RAD51C, RAD51D, STK11, TP53, XRCC2). As shown in Fig. 3a, this kit correctly detected the $c .68 \_69 \mathrm{del}$ variant (showing a variant allele frequency of about $8 \%$ ). In addition, NGS data provides also information regarding part of the $B R C A 1$ intron 2, including the $c .80+48 \_c .80+49$ site. Analyzing NGS results for this region (Fig. 3), a WT sequence was observed highlighting that the WT allele had been preferentially sequenced and confirming that the vector insertion region is wide extending beyond the $c .80+48 \_c .80+49$ site, as already assumed after performing Sanger sequencing.

\section{Confirmation of the c.68_69del variant via by an orthogonal method: a targeted Sanger sequencing}

In order to confirm the presence of the BRCA1 c.68_69del variant via Sanger sequencing, we designed an ad hoc PCR primer pair that ensured the amplification of the mutated c.68_69del allele. The antisense primer was designed to pair in the exon/intron junction of the $B R C A 1$ exon 2, before the c. $80+48 \_c .80+49$ site generating a small fragment, compatible with DNA quality from FFPE material (Fig. 2). As shown in Fig. 4, this strategy allowed us to correctly identify the $c .68 \_69 d e l$ variant in the EQA sample.

\section{Concluding remarks}

During an EQA program from EMQN for $t B R C A$ testing, our MGDUnit failed to identify the somatic BRCA1 c.68_69del variant. All laboratories that used the Deyvser $B R C A$ kit obtained the same result. The EMQN did not assign the category of poor performance to any laboratory that failed to detect the $c .68 \_69 \mathrm{del}$ variant using this kit. They acknowledged that the material used contained an artificial engineering scar that caused mis-priming of a PCR primer. We decided to explore this result using alternative methods

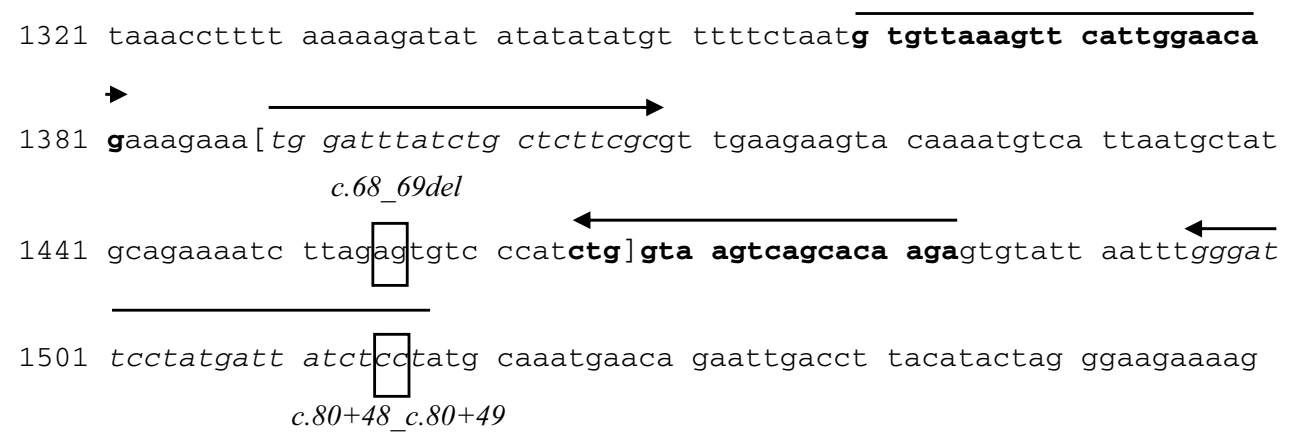

Fig. 2 The BRCA1 sequence surrounding the region of interest (LRG_292t1) is showed, with the exon 2 boundaries reported in bracket. The declared BRCAI variant c.68_69del and the insertion site $c .80+48 \_c .80+49$ are also depicted (boxes). The primer pair designed for the first Sanger se-quencing reaction are reported in italic as: forward: 5'-TGGATTTATCTG CTCTTCGC-3'; reverse:
5'-AGGAGATAATCATAGGAATCCC-3'. The primer pair used in the second Sanger sequencing reaction are reported in bold as: forward: 5'-GTGTTAAAGTTCATTGGAACAG-3'; reverse: 5'-TCT TGT GCT GAC TTA CCAG-3'. The latter allow the correct identification of the BRCA1 variant. The primers were designed by Primers 3 software (http://primer3.ut.ee/) 
a

GENES SNVS/INDELS CNVS FUSIONS WARNINGS

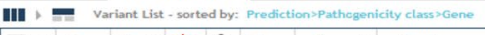

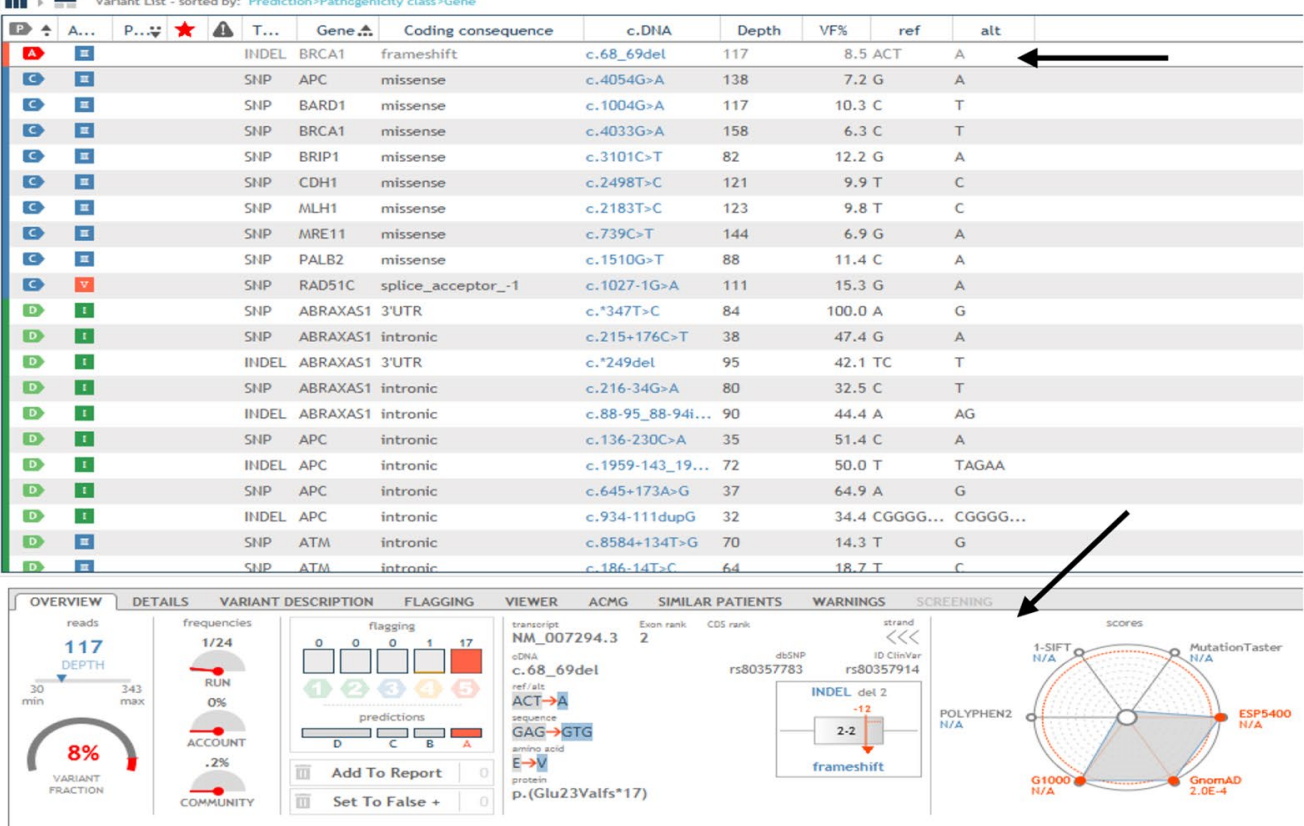

b

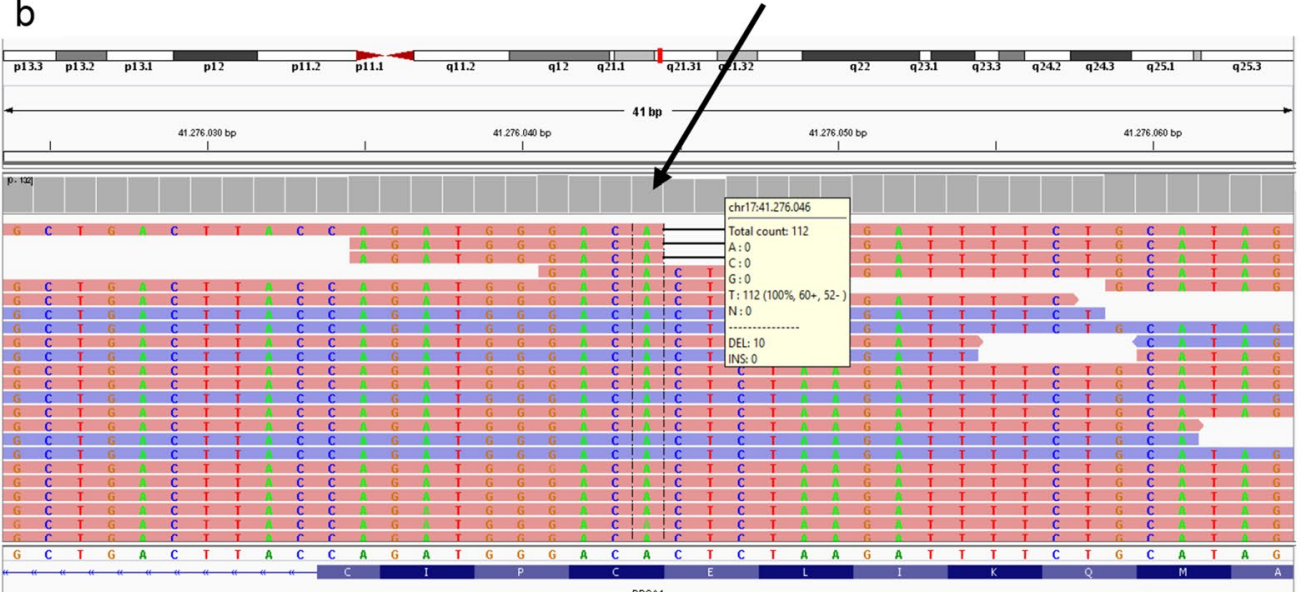

C
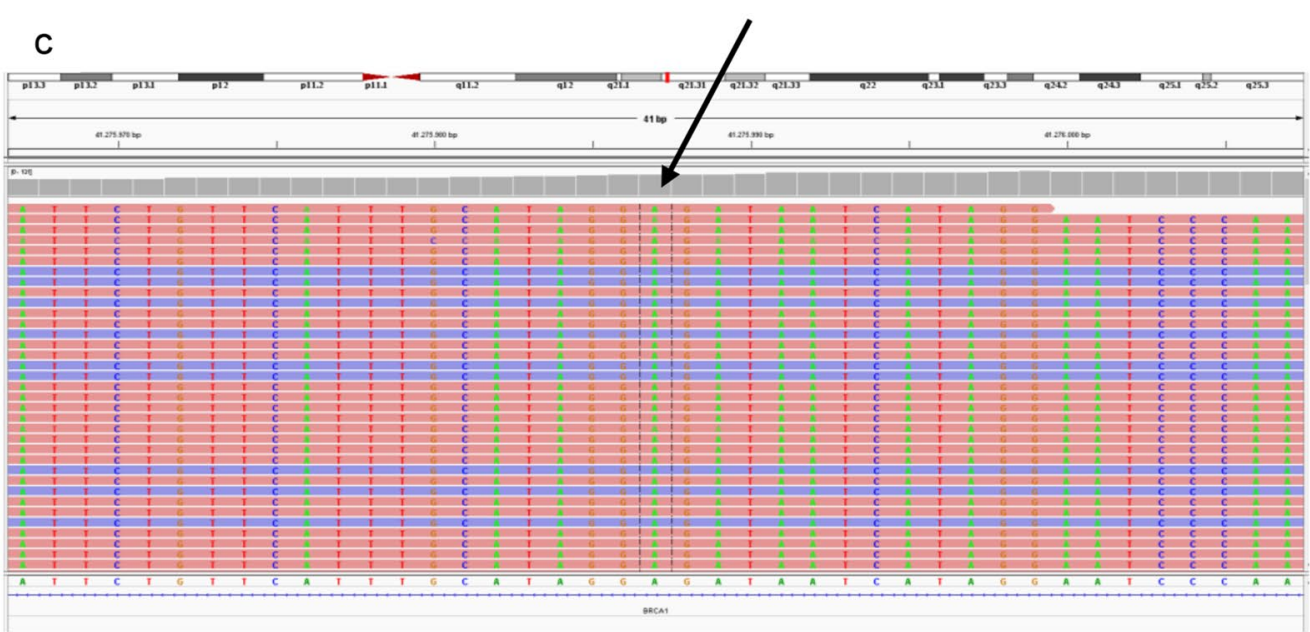
4Fig. 3 a The analysis performed by using the Sophia Genetics DDM software v3.4.0-4.6.2 (Sophia Genetics, Saint-Sulpice, Switzerland) for the BRCA1 c.68_69del (rs80357914) variant is reported, together with the associated variant information (black arrows). Integrative Genomics Viewer (IGV) visualizations of the BAM files obtained by using the Hereditary Cancer Solution CE-IVD kit (SOPHiA Genetics, Saint-Sulpice, Switzerland) are shown to highlight; b the coverage result and the reads data associated to the BRCA1 c.68_69del variant (black arrow); $\mathbf{c}$ the wild-type sequence obtained for the BRCAl region that includes the insertion site c.80+48_c.80+49 (black arrow). This evidence suggests that both amplification and sequencing involved only the wild-type allele

encompassing different PCR/NGS approaches in addition to the traditional Sanger sequencing technique. Firstly, an attempt using Sanger sequencing was made to identify the vector insertion region without success. Secondly, a different PCR/NGS method was used allowing to correctly identify the EMQN variant declared. Third, we confirmed the presence of the EMQN c.68_69del variant using a targeted Sanger sequencing designing $a d$ hoc an PCR/sequencing primer pair.

Sequencing of $B R C A$ genes is the prominent approach for routine screening of genomic variations in ovary, breast, pancreas and prostate cancer patients $[9,10]$. Participation to EQA allows monitoring of laboratory performance in an inter-laboratory level. In addition, EQA participation is an integral part of the quality framework of diagnostic laboratories, required by the International Organization for Standardization (ISO 15,189) [11].

A crucial aspect of EQA is the nature of the external source of quality control material. The ideal EQA sample should be obtained from clinical specimens, most closely representing real testing in a clinical laboratory [2]. However, the use of FFPE clinical tissue samples for large-scale formal EQA studies is nearly impossible due to the limited amount of tissue samples available from a given patient. In addition, the fixation process usually yields degraded DNA and sequence artifacts are frequently detected. Consequently, appropriate quantities of high-quality and stable FFPE clinical EQA samples are difficult to obtain [2]. In light of these critical issues, artificial samples, engineered to contain variants of interest, are often used in EQA schemes [4]. Here, we underlined that the DNA sequences flanking the mutations of interest in the artificial fragment must be identical to the in vivo genomic DNA sequences. In fact, this feature should allow to confirm the NGS results using a Sanger sequencing approach. In this study, only ad hoc Sanger sequencing allowed confirming the EMQN NGS results.

Moreover, as here described, some anecdotal evidences reported in literature indicate that NGS could not always optimally perform with DNA isolated using some external source [2] and errors, commonly associated to the assay design, could occur. ADO is a common phenomenon that reduces the efficiency of PCR-based targeted sequencing. It was first described as a "partial amplification failure" causing a potential source of misdiagnosis for both dominant and recessive diseases [12]. The ADO phenomenon involves selective allele amplification during the PCR process. The presence of single nucleotide variants in primer binding sites may lead to the complete or partial lack of amplification of the single allele, while the second may "drop" out during the PCR. Generally, the amplicon-based NGS strategy consists in multiplex PCR producing overlapping amplicons and providing a full and uniform coverage of all exons and exon/ intron junctions of genes. This method assures a higher coverage of insertions/deletions and also an accurate analysis of copy number variants. However, the ADO remains one of the most relevant technical limitation of different PCRbased methods, as depicted elsewhere [13]. Moreover, several cases of unrecognized polymorphic variants located in the binding sites of the amplification primer that preclude the detection of specific mutations, are reported in literature. For example, one EQA scheme for Cystic Fibrosis evidenced that a laboratory-developed test could not accurately detect the $c .621+1 G>T$ mutation declared in the quality assessment [14]. Similar evidence was reported for BRCA testing [15] and for hereditary hemochromatosis [16]. In all these cases, the involved laboratories used the observed discrepancies as an opportunity to review their analytical process. The experienced ADO caused by the mutagenesis method using the Devyser BRCA kit reinforce the need of a careful optimization of the NGS workflow. In our Devyser $B R C A$ NGS diagnostic workflow, the monitoring of ADO events is obtained via the identification of a strong and deep deletion signal obtained in the NGS copy number variant evaluation involving an amplicon among all the overlapping amplicons covering one exon. In this type of suggestive situation, we perform an alternative NGS test using a capture-based method, as described for the purpose of this paper.

Considering that the library preparation kit used in our molecular workflow is an amplicon-based type, at first we suspected the occurring of an ADO event. However, in the light of what has been reported, the ADO turns out to be exclusively dependent on the artificial nature of the external EMQN sample. Our efforts to understand the unexpected EQA scheme result, underlined the educational aspect of the EQA process, which generally helps laboratories to detect errors in their protocols and adopt corrective strategies improving the molecular assay outcome.

\section{Summary}

In this paper, we focused on an EMQN scheme used for the EQA of somatic variant detection in $B R C A$ genes. In this context, our MGDUnit experienced a failure in EQA performance. However, a careful and critical evaluation of 


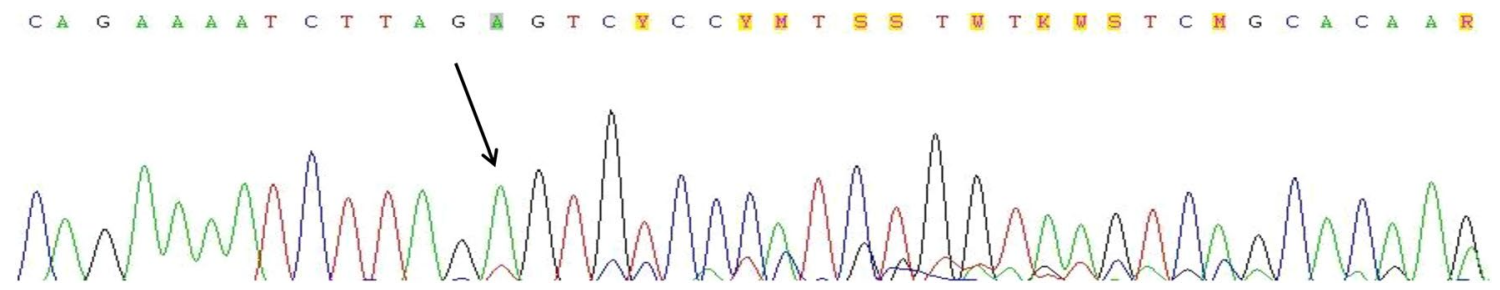

Fig. 4 Sanger sequencing result of the $B R C A 1$ exon 2 region surrounding the EMQN declared variant (LRG_292t1). The presence of the BRCA1 c.68_69del variant at low VAF was depicted with a clear shift of the nucleotide sequence from the position $c .68 \mathrm{~A}$ (black arrow). Sanger sequencing was performed with the Applied Biosystems 3500 Genetic Analyzer (Life Technologies, Carlsbad, CA, USA) this error help us to better understand if corrective actions, as redesigning tests or adjustment of methods, are needed.

We underlined that the adoption of complex testing methods, as NGS, should require implementation or optimization of the actual EQA design. Ideally, major attention should be paid to the plethora of amplification and NGS approaches available, ensuring that the success of an EQA is independent from the type of NGS or library preparation approach used.

New EQA approaches could be useful to ensure the continued quality in case of advanced and complex technologies. For example, a new prospective emerges from EQA schemes that use FFPE samples derived from untransformed cells modified using the CRISPR/Cas9 system without dramatically altering the original DNA sequence [2].

In conclusion, an appropriate EQA material could be useful to assure high-quality NGS testing in clinical laboratory settings. With this regard, each laboratory should take part to EQA schemes in order to critically analyze and improve the quality of own work. We would like to point out that a critical approach is always recommended to test the laboratory skills and abilities.

Acknowledgements We would like to thank Franziska M. Lohmeyer, $\mathrm{PhD}$, Fondazione Policlinico Universitario A. Gemelli IRCCS, for her support revising our manuscript.

Author contributions EDP and AM contributed conception and design of the study. EDP and MEO performed the experiments. AM, PC and $\mathrm{CS}$ analyzed data. All authors contributed to manuscript revision, read, and approved the submitted version.

Funding Open access funding provided by Università Cattolica del Sacro Cuore within the CRUI-CARE Agreement.

\section{Declarations}

Conflict of interest The authors declare that they have no conflict of interest.
Ethical approval This study complied with the Ethical Principles for Medical Research Involving Human Subjects according to the World Medical Association Declaration of Helsinki and was certified by the Committee of the Applicable Institution of the Fondazione Policlinico Universitario Agostino Gemelli IRCCS, Rome.

Consent to publish All authors have given their consent to participate in this report and submit it to Molecular Biology Reports.

Open Access This article is licensed under a Creative Commons Attribution 4.0 International License, which permits use, sharing, adaptation, distribution and reproduction in any medium or format, as long as you give appropriate credit to the original author(s) and the source, provide a link to the Creative Commons licence, and indicate if changes were made. The images or other third party material in this article are included in the article's Creative Commons licence, unless indicated otherwise in a credit line to the material. If material is not included in the article's Creative Commons licence and your intended use is not permitted by statutory regulation or exceeds the permitted use, you will need to obtain permission directly from the copyright holder. To view a copy of this licence, visit http://creativecommons.org/licenses/by/4.0/.

\section{References}

1. Schuster SC (2008) Next-generation sequencing transforms today's biology. Nat Methods 5:16-18. https://doi.org/10.1038/ nmeth 1156

2. Kalman LV, Lubin IM, Barker S, Barker S, du Sart D, Elles R, Grody W, Pazzagli W, Richards M, Schrijver S, Zehnbauer I B (2013) Current landscape and new paradigms of proficiency testing and external quality assessment for molecular genetics. Arch Pathol Lab Med 137:983-988. https://doi.org/10.5858/arpa. 2012-0311-RA

3. Payne DA, Russomando G, Linder MW, Baluchova K, Ashavaid T, Steimer W, Ahmad-Nejad P, IFCC Committee for Molecular Diagnostics (C-MD) (2020) External quality assessment (EQA) and alternative assessment procedures (AAPs) in molecular diagnostics: findings of an international survey. Clin Chem Lab Med 59:301-306. https://doi.org/10.1515/cclm-2020-0101

4. Lin G, Zhang K, Han Y, Li J (2017) Quality control materials for pharmacogenomic testing in the clinic. Clin Chem Lab Med 55:926-933. https://doi.org/10.1515/cclm-2016-0755

5. Marchetti C, Minucci A, D'Indinosante M, Ergasti R, Arcieri M, Capoluongo ED, Pietragalla A, Caricato C, Scambia G, Fagotti A (2020) Feasibility of tumor testing for BRCA status in high-grade 
serous ovarian cancer using fresh-frozen tissue based approach. Gynecol Oncol 158:740-746. https://doi.org/10.1016/j.ygyno. 2020.06.479

6. European Molecular Genetics Quality Network. The European Molecular Genetics Quality Network Web site. https://doi.org/ http://www.emqn.org/emqn/Home. Accessed Giu 24, 2021

7. De Paolis E, De Bonis M, Concolino P, Piermattei A, Fagotti A, Urbani A, Scambia G, Minucci A, Capoluongo E (2021) Droplet digital PCR for large genomic rearrangements detection: a promising strategy in tissue BRCA1 testing. Clin Chim Acta 513:17-24. https://doi.org/10.1016/j.cca.2020.12.001

8. Concolino P, Mello E, Minucci A, Santonocito C, Scambia G, Giardina B, Capoluongo E (2014) Advanced tools for BRCA1/2 mutational screening: comparison between two methods for large genomic rearrangements (LGRs) detection. Clin Chem Lab Med 52(8):1119-1127. https://doi.org/10.1515/cclm-2013-1114

9. Minucci A, Scambia G, Santonocito C, Concolino P, Urbani A (2020) BRCA testing in a genomic diagnostics referral center during the COVID-19 pandemic. Mol Biol Rep 47:4857-4860. https://doi.org/10.1007/s11033-020-05479-3

10. Wong RSJ, Lee SC (2020) BRCA sequencing of tumors: understanding its implications in the oncology community. Chin Clin Oncol 9:66. https://doi.org/10.21037/cco-19-198

11. Schneider F, Maurer C, Friedberg RC (2017) International Organization for Standardization (ISO) 15189. Ann Lab Med 37:365370. https://doi.org/10.3343/alm.2017.37.5.365

12. Shestak AG, Bukaeva AA, Saber S, Zaklyazminskaya EV (2021) Allelic dropout is a common phenomenon that reduces the diagnostic yield of PCR-Based sequencing of targeted gene panels. Front Genet 12:620337. https://doi.org/10.3389/fgene.2021. 620337

13. Concolino P, Rizza R, Mignone $F$ et al. A comprehensive BRCA1/2 NGS pipeline for an immediate Copy Number Variation (CNV) detection in breast and ovarian cancer molecular diagnosis. Clin Chim Acta. 480:173-179. https://doi.org/10.1016/j.cca.2018. 02.012

14. Dequeker E, Cassiman JJ (1998) Evaluation of CFTR gene mutation testing methods in 136 diagnostic laboratories: report of a large European external quality assessment. Eur J Hum Genet 6:165-175. https://doi.org/10.1038/sj.ejhg.5200195

15. Ramsden SC, Deans Z, Robinson DO, Mountford R, Sistermans EA, Grody WW, McQuaid S, Patton SJ, Stenhouse SA (2006) Monitoring standards for molecular genetic testing in the United Kingdom, the Netherlands, and Ireland. Genet Test 10:147-156. https://doi.org/10.1089/gte.2006.10.147

16. Noll WW, Belloni DR, Stenzel TT, Grody WW (1999) Polymorphism in intron 4 of HFE does not compromise haemochromatosis mutation results. Nat Genet 23:271-272. https://doi.org/10.1038/ 15722

Publisher's Note Springer Nature remains neutral with regard to jurisdictional claims in published maps and institutional affiliations. 